\title{
Differentiation and localization of targets using infrared sensors
}

\author{
Tayfun Aytaç, Billur Barshan* \\ Department of Electrical Engineering, Bilkent University, TR-06533 Bilkent, Ankara, Turkey
}

Received 22 April 2002; accepted 27 June 2002

\begin{abstract}
This study investigates the use of low-cost infrared emitters and detectors in the differentiation and localization of commonly encountered features or targets in indoor environments, such as planes, corners, edges, and cylinders. The intensity readings obtained with such systems are highly dependent on target location and properties in a way which cannot be represented in a simple manner, making the differentiation and localization process difficult. In this paper, we propose the use of angular intensity scans and present an algorithm to process them. This approach can determine the target type independent of its position. Once the target type is identified, its position can also be estimated. The method is verified experimentally. An average correct classification rate of $97 \%$ over all target types is achieved and targets are localized within absolute range and azimuth errors of $0.8 \mathrm{~cm}$ and $1.6^{\circ}$, respectively. The method demonstrated shows that simple infrared sensors, when coupled with appropriate processing, can be used to extract a significantly greater amount of information than that which they are commonly employed for. (c) 2002 Elsevier Science B.V. All rights reserved.
\end{abstract}

Keywords: Pattern recognition and feature extraction; Position estimation; Infrared sensors

\section{Introduction}

Target differentiation and localization is of considerable interest for intelligent systems where there is need to identify targets and their positions for autonomous operation. In this paper, we consider the use of a simple infrared sensing system consisting of one emitter and one detector for this purpose. These devices are inexpensive, prac-

\footnotetext{
${ }^{*}$ Corresponding author. Tel.: +90-312-290-2161; fax: +90312-266-4192.

E-mail addresses: taytac@ee.bilkent.edu.tr (T. Aytac), billur@ee.bilkent.edu.tr (B. Barshan).
}

tical and widely available. The emitted light is reflected from the target and its intensity is measured at the detector. However, it is often not possible to make reliable distance estimates based on the value of a single intensity return because the return depends on both the geometry and other properties of the reflecting target. Likewise, the properties of the target cannot be deduced from simple intensity returns without knowing its distance and angular location. In this paper, we propose a scanning technique and algorithm that can determine the type of the target in a manner which is invariant to its location. Once the target type is determined, its position $(r, \theta)$ can also be estimated. Our results 
show that by properly processing data obtained from simple infrared sensors, it is possible to extract a significantly greater amount of information than that which such devices are commonly employed for.

The method we propose is scalable in the sense that the accuracy can be increased by increasing the number of reference scans without increasing the computational complexity of the differentiation and localization process.

Most work on pattern recognition involving infrared deals with recognition or detection of features or targets in conventional two-dimensional images. Examples of work in this category include face identification [1], automatic target recognition [2], target tracking [3], automatic vehicle detection [4], remote sensing [5], detection and identification of targets in background clutter $[6,7]$, and automated terrain analysis [8]. We note that the position-invariant pattern recognition and position estimation achieved in this paper is different from such operations performed on conventional images $[9,10]$ in that here we work not on direct "photographic" images of the targets obtained by some kind of imaging system, but rather on angular intensity scans obtained by rotating a point sensor. The targets we differentiate are not patterns in a two-dimensional image whose coordinates we try to determine, but rather objects in space, exhibiting depth, whose position with respect to the sensing system we need to estimate. As such, position-invariant differentiation and localization is achieved with an approach quite different than those employed in invariant pattern recognition and localization in conventional images [11-18].

Infrared sensors are used in robotics and automation, process control, remote sensing, and safety and security systems. More specifically, they have been used in simple object and proximity detection [19], counting [20], distance and depth monitoring [21], floor sensing, position control [22], and obstacle/collision avoidance [23]. Infrared sensors are used in door detection and mapping of openings in walls [24], as well as monitoring doors/ windows of buildings and vehicles, and "light curtains" for protecting an area. In [25], the properties of a planar surface at a known distance have been determined using the Phong illumination model, and using this information, the infrared sensor employed has been modeled as an accurate range finder for surfaces at short ranges. Refs. [26-28] deal with optical determination of depth information. Hashimoto et al. [29] describes a passive infrared sensing system which identifies the locations of the people in a room. Infrared sensors have also been used for automated sorting of waste objects made of different materials [30]. However, to the best of our knowledge, no attempt has been made to differentiate and estimate the position of several kinds of targets using infrared sensors. In this paper, we show that by appropriate processing and application of pattern recognition techniques, it is possible to achieve these objectives. Our results show that it is possible to extract a significantly greater amount of information from simple optical sensors than which they are commonly employed for (e.g., the emitter and detector pair employed in this study is marketed as a simple proximity switch).

\section{Target differentiation and localization}

The infrared sensor [31] used in this study consists of an emitter and detector and works with 20-28 V dc input voltage, and provides analog output voltage proportional to the measured intensity reflected off the target. The detector window is covered with an infrared filter to minimize the effect of ambient light on the intensity measurements. Indeed, when the emitter is turned off, the detector reading is essentially zero. The sensitivity of the device can be adjusted with a potentiometer to set the operating range of the system.

The targets employed in this study are plane, $90^{\circ}$ corner, $90^{\circ}$ edge, and a cylinder of radius 4.8 $\mathrm{cm}$, whose cross-sections are given in Fig. 1. They are made of wood, each with a height of $120 \mathrm{~cm}$. Our method is based on angularly scanning each target over a certain angular range. The infrared sensor is mounted on a $12 \mathrm{in.} \mathrm{rotary}$ table (Fig. 2) [32] to obtain angular scans from these target primitives. Reference data sets are collected for each target with $2.5 \mathrm{~cm}$ distance increments, ranging from $15 \mathrm{~cm}$ to the maximum detectable 

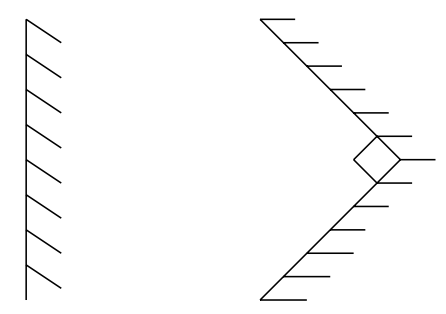

corner

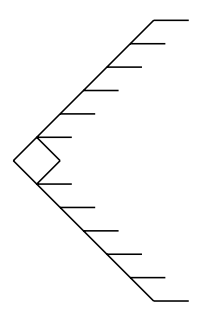

edge

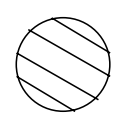

cylinder

Fig. 1. Target primitives used in this study.

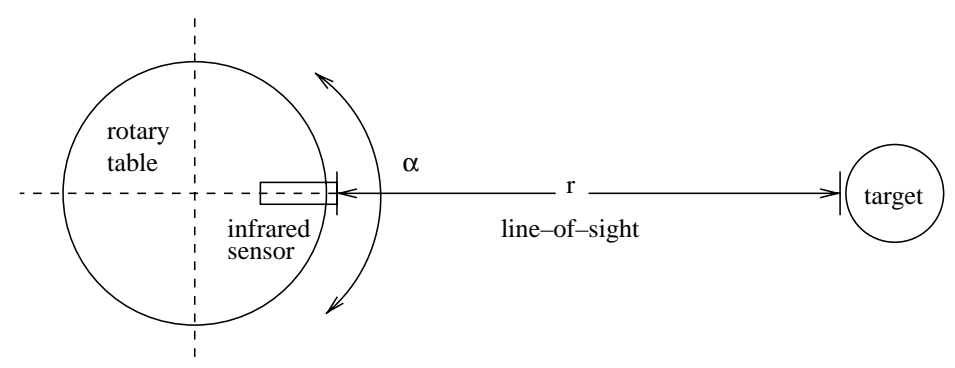

Fig. 2. Top view of the experimental setup used in target differentiation and localization. The emitter and detector windows are circular with $8 \mathrm{~mm}$ diameter and center-to-center separation $12 \mathrm{~mm}$. (The emitter is above the detector.) Both the scan angle $\alpha$ and the target azimuth $\theta$ are measured counter-clockwise from the horizontal axis.

range of each target, at $\theta=0^{\circ}$. The output signal is processed using an 8-bit microprocessor compatible $\mathrm{A} / \mathrm{D}$ converter chip having a conversion time of $100 \mu \mathrm{s}$.

The resulting reference scans for plane, cylinder, edge and corner are shown in Figs. 3-6, respectively. The intensity scans are $\theta$-invariant but not $r$-invariant; changes in $r$ do not result in any simple scaling. As we will see, these scans contain sufficient information to identify and localize the different target types with a good degree of accuracy. Fig. 6 shows the distinctive double-humped scan pattern for the corner target (this doublehumped pattern can be interpreted by thinking of the corner in terms of its two orthogonal constituent planes). As can be guessed from these figures, the greatest difficulty is encountered in differentiating cylinders and edges which have the most similar intensity patterns. Notice that the return signal intensities saturate at an intensity corresponding to $10.7 \mathrm{~V}$ output voltage.

We now describe how to determine the target type and position of an arbitrarily located target whose intensity scan has been observed. First, we check whether the observed scan $I(\alpha)$ exhibits saturation or not. This situation is treated separately as will be explained later in Section 2.3. A corner scan is considered saturated when its central intensity enters the saturation region, not the humps, since it is the former value which is critical for our method below.

We start by determining the target type. Unfortunately, direct comparison with the corresponding curves in Figs. 3-6 is not possible since we do not yet know the distance of the target, and comparing with all the curves at all distances would be computationally very expensive. Therefore, we exploit the fact that the successive curves in Figs. 36 exhibit a monotonic dependence on distance. Furthermore, when an observed scan is compared to the several successive curves in any of Figs. 3-6, the two measures of difference between them described in Sections 2.1 and 2.2 below also exhibit a monotonic fall and rise around a single minimum. Therefore, we are assured that we will not be settling at a suboptimal point if we compare the 


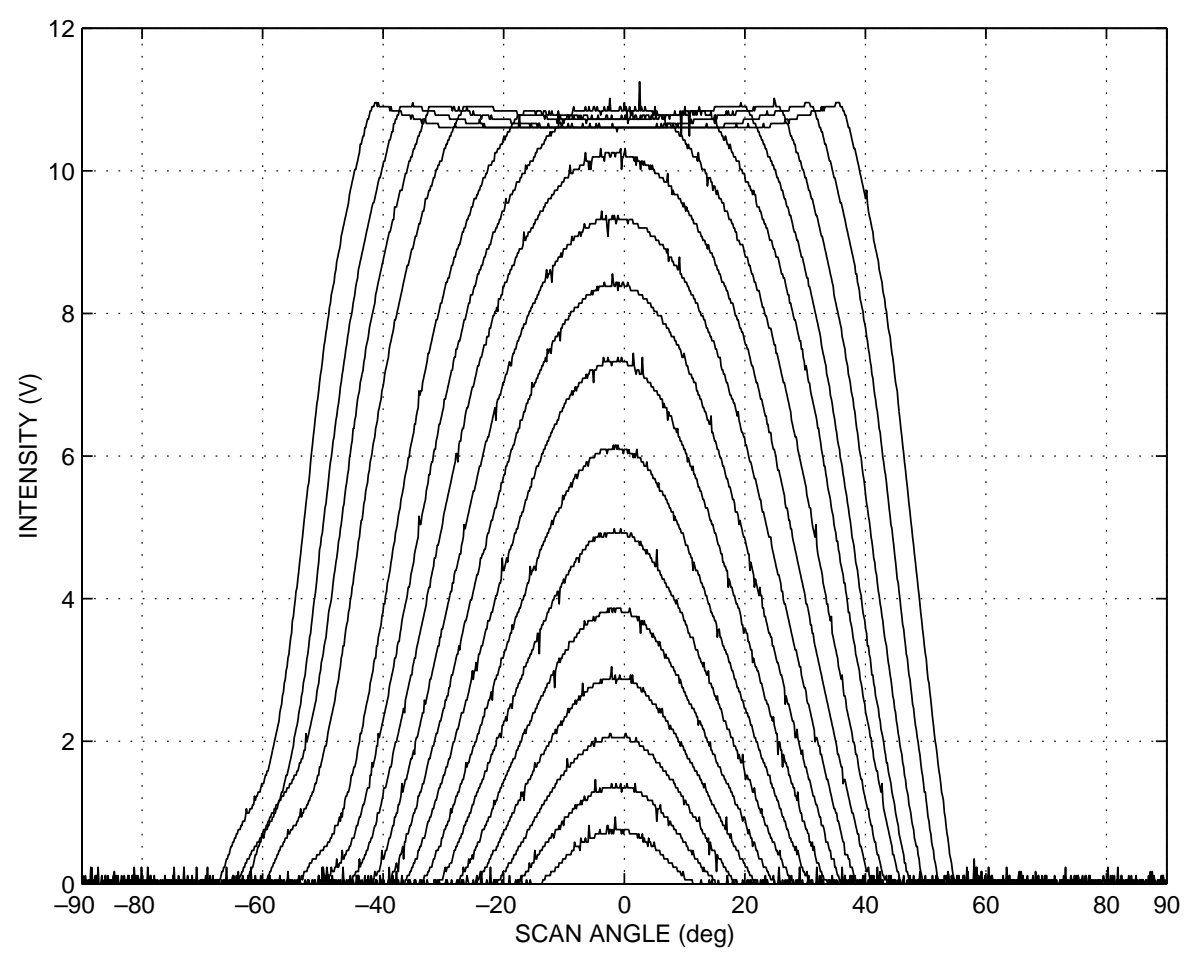

Fig. 3. Intensity scans for planes at different distances.

observed scan not with all scans at all distances but only with the four scans (one for each target type) whose central intensities are closest to that of the observed scan. Therefore, for unsaturated scans, only four comparisons need to be made. This remains the case even if the $2.5 \mathrm{~cm}$ increments are reduced to smaller values. This has the advantage that the accuracy of the system can be increased without increasing the cost of computation (although a greater number of scans do have to be stored). As a test, we also ran a version of the method where eight comparisons were made using the scans with the nearest central intensities both above and below the observed central intensity, and also using all of the scans shown in Figs. 3-6. These computationally more expensive approaches, exceedingly more so in the latter case, did not improve the result with respect to comparison with only four scans. In fact, in the matched filtering case discussed in Section 2.2, the results are even somewhat better when four scans are used, due to the fact that this systematic elimination of a priori suboptimal scans eliminates the small possibility that they will mistakingly be chosen as the best matching scan due to noise and other errors.

Two alternative approaches are employed in performing the four comparisons. These are discussed below in the following two sections.

\subsection{Least-squares approach}

First, we estimate the angular position of the target as follows: Assuming the observed scan pattern is not saturated, we check if it has two humps or not. If so, it is a corner and we find the angular location of the dip in the middle of the two humps and the corresponding intensity value. If not, we find the angular location of the maximum, denoted $\theta_{\mathrm{MAX}}$, and again the corresponding intensity value. These angular values can be directly taken as estimates of the angular position of the target. Alternatively, the angular position can be estimated by finding the center-of-gravity (COG) of the scan as follows 


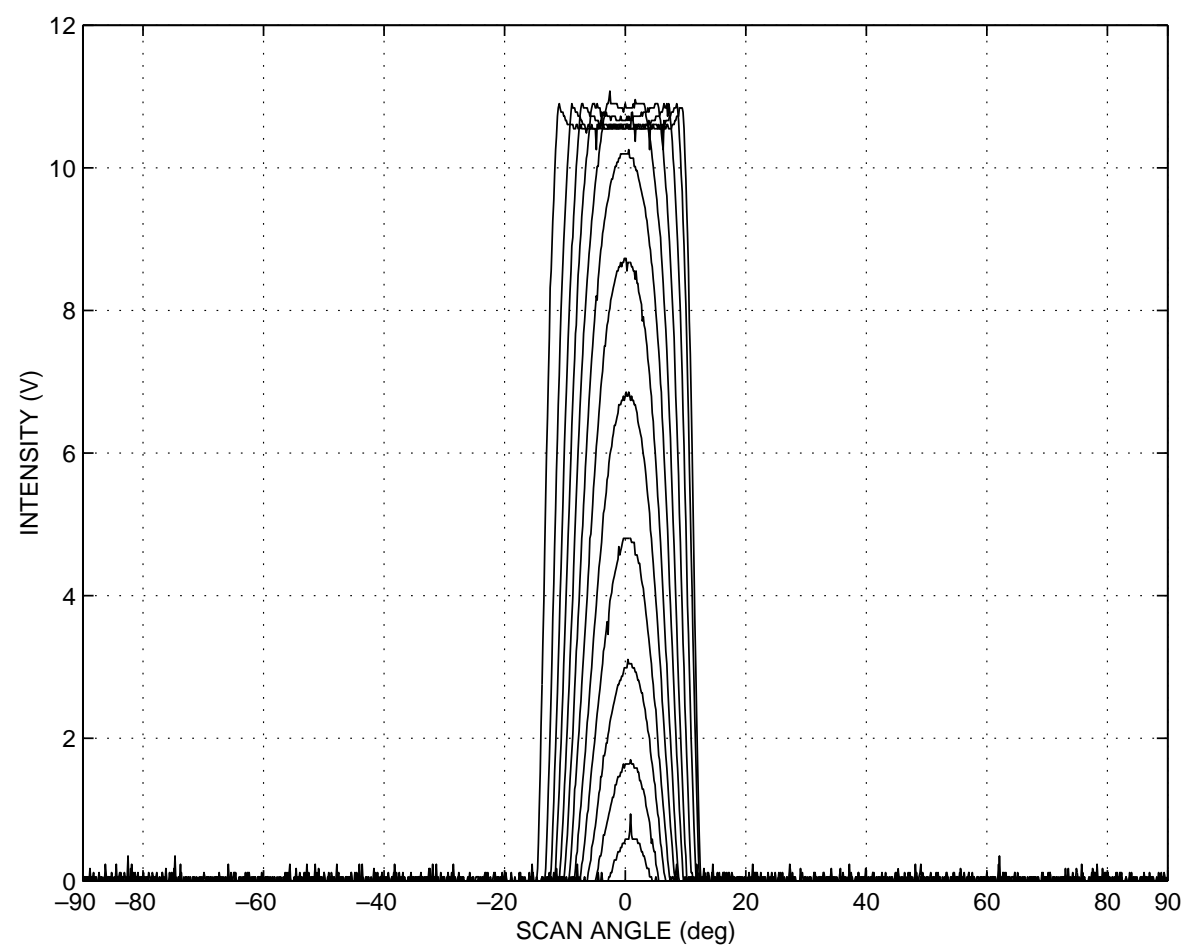

Fig. 4. Intensity scans for cylinders at different distances.

$\theta_{\mathrm{COG}}=\frac{\sum_{i=1}^{n} \alpha_{i} I\left(\alpha_{i}\right)}{\sum_{i=1}^{n} I\left(\alpha_{i}\right)}$

where $n$ is the number of samples in the angular scan. Ideally, these estimates would be equal, but in practice they differ by a small amount. We will consider the use of both alternatives when tabulating our results. From now on, we will refer to either estimate as the "center angle" of the scan.

Plots of the intensity at the center angle of each scan in Figs. 3-6 as a function of the distance at which that scan was obtained, play an important part in our method. Fig. 7 shows these plots for the maximum intensity (central dip intensity for corner) case.

In this approach, we compare the intensity scan of the observed target with the four reference scans by computing their least-squares differences after aligning their centers with each other. Since the squared difference is sensitive even to multiplicative factors which are close to unity, we have employed an interpolated reference scan obtained by linearly interpolating between the two consecutive scans whose central intensities are just above and just below the observed scan. The mean-square difference between the observed scan and the four interpolated scans, one for each possible target type, is computed as follows

$\mathscr{E}_{j}=\sum_{i=1}^{n}\left[I\left(\alpha_{i}-\alpha_{\text {align }}\right)-I_{j}\left(\alpha_{i}\right)\right]^{2}$,

where $I_{j}, j=1,2,3,4$ denote the four interpolated scans. Here, $\alpha_{\text {align }}$ is the angular shift which is necessary to align both patterns. The target type resulting in the smallest value of $\mathscr{E}$ is declared as the observed target. Once the target type is determined, the range can be estimated by using linear interpolation on Fig. 7. Note that, this way, the accuracy of the method is not limited by the $2.5 \mathrm{~cm}$ spacing used in collecting the reference scans.

\subsection{Matched filtering approach}

As an alternative, we have also considered the use of matched filtering [33] to compare the 


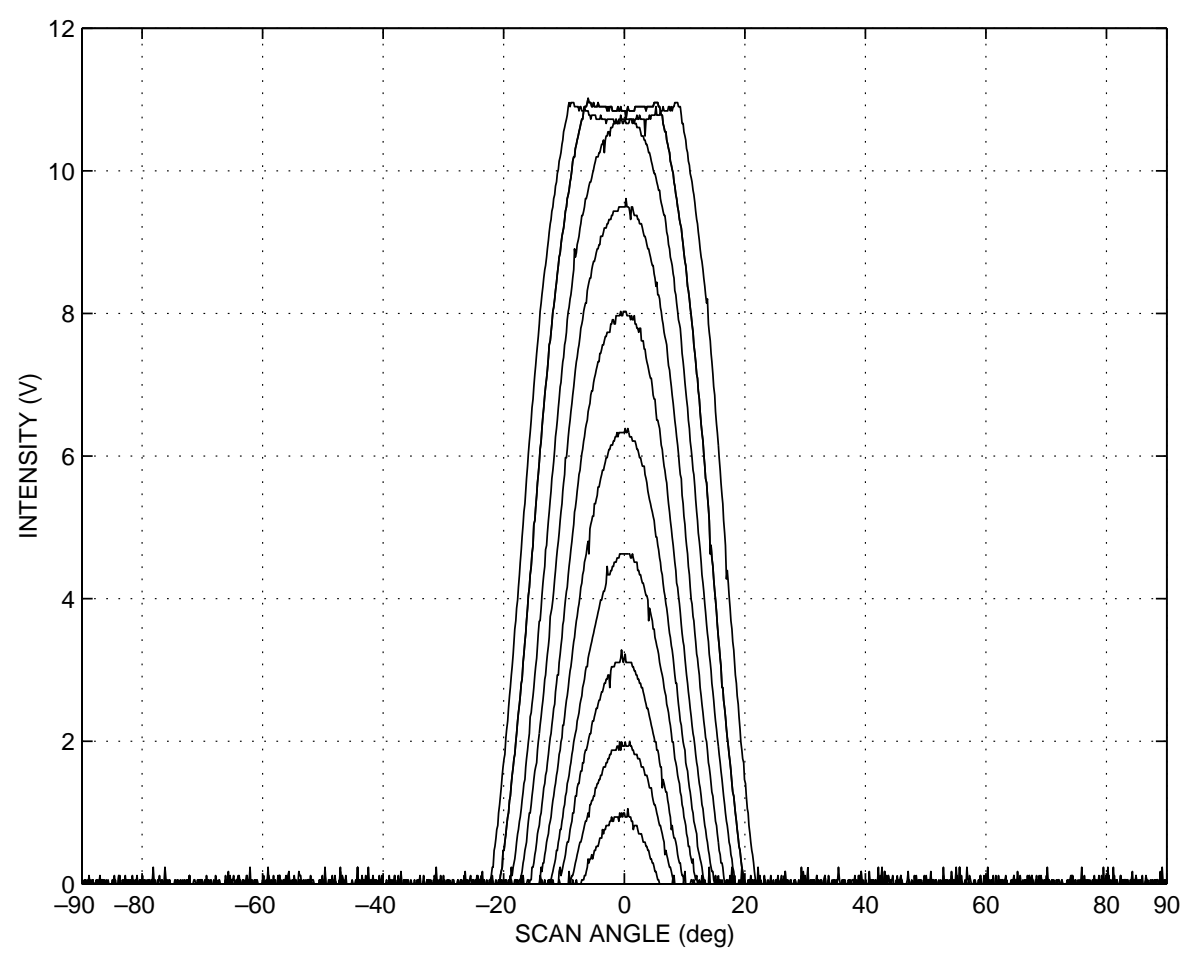

Fig. 5. Intensity scans for edges at different distances.

observed and reference scans. The output of the matched filter is the cross-correlation between the observed intensity scan and the $j$ th reference scan normalized by the square root of its total energy

$y_{j}(l)=\frac{\sum_{k} I\left(\alpha_{k}\right) I_{j}\left(\alpha_{k-l}\right)}{\sqrt{\sum_{k}\left[I_{j}\left(\alpha_{k}\right)\right]^{2}}}$.

The target type corresponding to the maximum cross-correlation peak is declared as the correct target type, and the angular position of the correlation peak directly provides an estimate of the azimuth angle of the target. Then, the distance is estimated by using linear interpolation on Fig. 7 with the intensity value at the azimuth estimate.

\subsection{Saturated scans}

If saturation is detected in the observed scan, special treatment is necessary. In the least-squares approach, the mean-square difference between the aligned observed scan and all the saturated reference scans are computed and the target type with the minimum mean-square difference is chosen. The range estimate of the target is taken as the distance corresponding to the scan resulting in the minimum mean-square difference. Similarly, for the matched filter, correlation between the observed scan and all the stored saturated reference scans is computed and the target type resulting in the highest correlation peak is selected. The range estimate is again taken as that of the best matching scan.

It should be noted that, in the saturated case, range estimation accuracy is limited by the $2.5 \mathrm{~cm}$ interval at which the reference scans were taken since interpolation is not possible. If this accuracy is not satisfactory, it can be improved by reducing the $2.5 \mathrm{~cm}$ intervals. We underline that the $2.5 \mathrm{~cm}$ interval does not limit the range estimation accuracy in the unsaturated case, where accurate interpolation is possible from Fig. 7. 


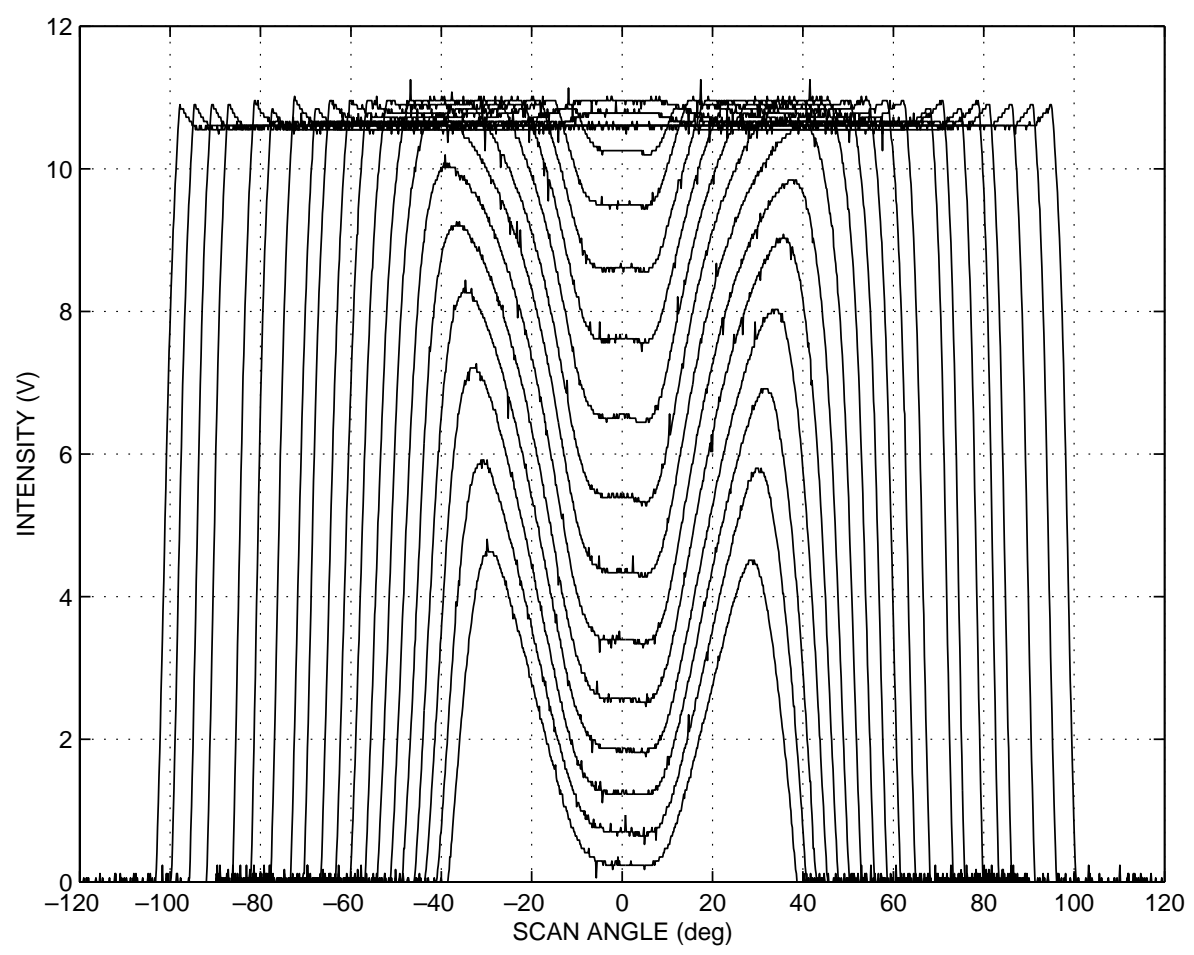

Fig. 6. Intensity scans for corners at different distances.

\section{Experimental verification and discussion}

In this section, we experimentally verify the proposed method by locating the targets at randomly selected distances $r$ and azimuth angles $\theta$ and collecting a total of 120 test scans. The targets are randomly located at azimuths varying from $-45^{\circ}$ to $45^{\circ}$ from $15 \mathrm{~cm}$ up to the maximum ranges in Figs. 3-6.

The results of least-squares based target differentiation are displayed in Tables 1 and 2 in the form of target confusion matrices. Table 1 gives the results obtained using the maximum (or the central dip for corner) intensity values, and Table 2 gives those obtained using the intensity value at the COG of the scans. The average accuracy over all target types can be found by summing the correct decisions given along the diagonal of the confusion matrix and dividing this sum by the total number of test trials (120). The average correct classification rates obtained by using the max/ dip and the COG variations of the least-squares approach are $93 \%$ and $89 \%$, respectively.

Matched filter differentiation results are presented in Table 3 . The average accuracy of differentiation over all target types is $97 \%$ which is better than that obtained with the least-squares approach. The matched filter correctly classifies planar targets as well as corners with an accuracy of $100 \%$.

As shown in the tables, corners are always correctly identified regardless of which method is used, due to their distinctive signature. Second best to corners are planes which are also usually correctly identified. Cylinders and edges are the most confused target types as we had expected from the similar nature of their intensity scans. Nearly all misclassified targets are located at far ranges where the return signal intensities are very weak.

The average absolute range and azimuth estimation errors for the different approaches are presented in Table 4 over all test targets. As seen in 


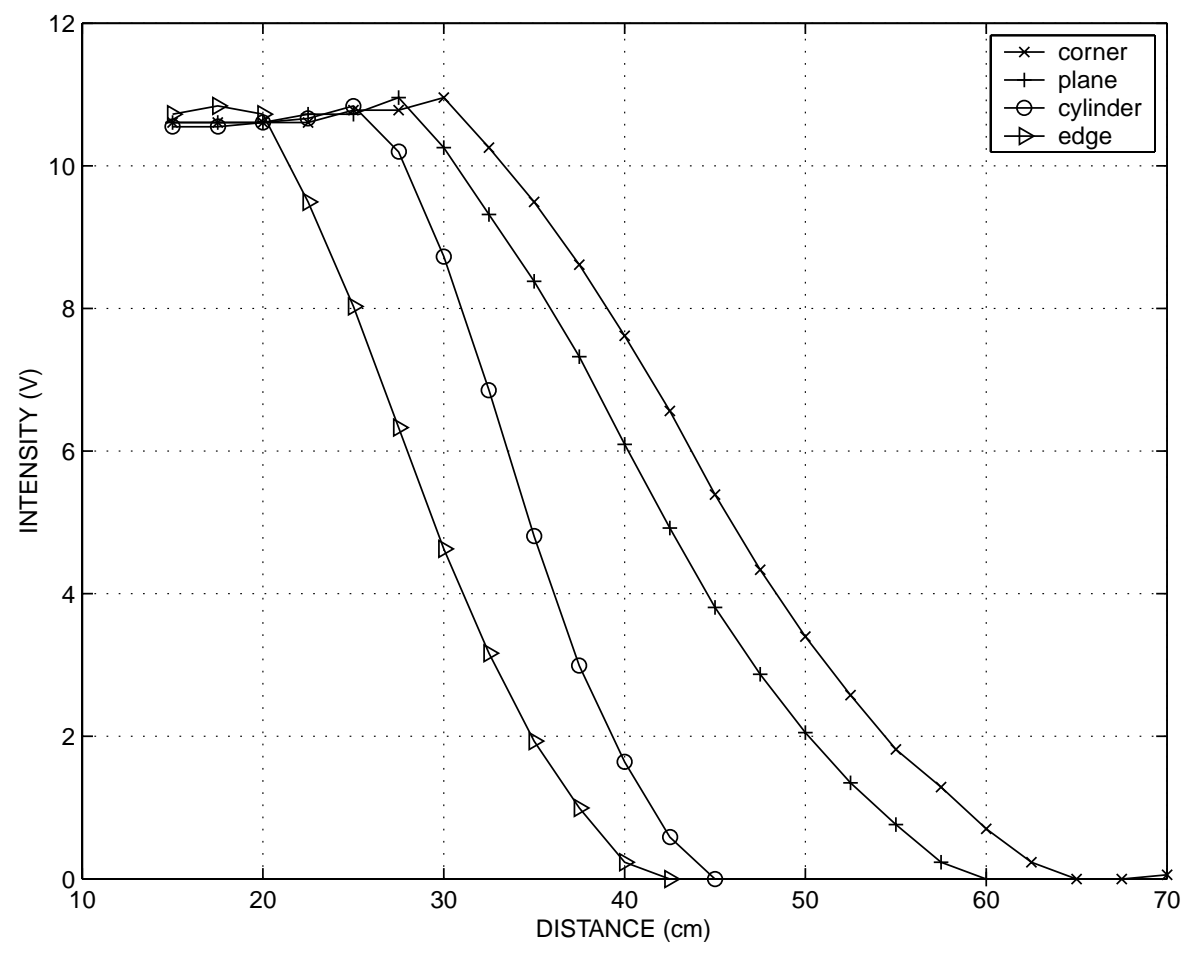

Fig. 7. Central intensity versus distance curves for the different targets.

Table 1

Target confusion matrix: least-squares based classification (max/dip variation)

\begin{tabular}{|c|c|c|c|c|c|}
\hline \multirow[t]{2}{*}{ Target } & \multicolumn{4}{|c|}{ Differentiation result } & \multirow[t]{2}{*}{ Total } \\
\hline & $\mathrm{P}$ & $\mathrm{CY}$ & $E$ & $\mathrm{C}$ & \\
\hline$P$ & 29 & - & 1 & - & 30 \\
\hline $\mathrm{CY}$ & 4 & 26 & - & - & 30 \\
\hline $\mathrm{E}$ & 1 & 3 & 26 & - & 30 \\
\hline $\mathrm{C}$ & - & - & - & 30 & 30 \\
\hline Total & 34 & 29 & 27 & 30 & 120 \\
\hline
\end{tabular}

P: plane, CY: cylinder, E: edge, C: corner.

Table 2

Target confusion matrix: least-squares based classification (COG variation)

\begin{tabular}{lccccc}
\hline Target & \multicolumn{2}{c}{ Differentiation result } & E & Total \\
\cline { 2 - 5 } & $\mathrm{P}$ & $\mathrm{CY}$ & - & $\mathrm{C}$ & 30 \\
\hline $\mathrm{P}$ & 30 & - & 2 & - & 30 \\
$\mathrm{CY}$ & 4 & 2 & -23 & - & 30 \\
$\mathrm{E}$ & 5 & - & - & 30 & 30 \\
C & - & 26 & 25 & 30 & 120 \\
Total & 39 & & & & \\
\hline
\end{tabular}


Table 3

Target confusion matrix: matched filter based classification

\begin{tabular}{|c|c|c|c|c|c|}
\hline \multirow[t]{2}{*}{ Target } & \multicolumn{4}{|c|}{ Differentiation result } & \multirow[t]{2}{*}{ Total } \\
\hline & $\mathrm{P}$ & $\mathrm{CY}$ & $\mathrm{E}$ & $\mathrm{C}$ & \\
\hline$P$ & 30 & - & - & - & 30 \\
\hline $\mathrm{CY}$ & - & 27 & 3 & - & 30 \\
\hline $\mathrm{E}$ & - & 1 & 29 & - & 30 \\
\hline $\mathrm{C}$ & - & - & - & 30 & 30 \\
\hline Total & 30 & 28 & 32 & 30 & 120 \\
\hline
\end{tabular}

Table 4

Absolute range and azimuth estimation errors over all test targets

\begin{tabular}{lllllll}
\hline Method & & $\mathrm{P}$ & $\mathrm{CY}$ & $\mathrm{E}$ & $\mathrm{C}$ & Average error \\
\hline Least squares & $r(\mathrm{~cm})$ & 1.0 & 1.8 & 1.1 & 0.7 & 1.2 \\
(max/dip) & $\theta(\mathrm{deg})$ & 4.1 & 1.7 & 2.3 & 5.7 & 3.5 \\
Least squares & $r(\mathrm{~cm})$ & 0.5 & 1.5 & 4.3 & 0.7 & 1.7 \\
(COG) & $\theta(\mathrm{deg})$ & 2.9 & 2.4 & 3.0 & 2.8 & 2.8 \\
Matched filter & $r(\mathrm{~cm})$ & 0.7 & 1.0 & 0.8 & 0.7 & 0.8 \\
& $\theta(\mathrm{deg})$ & 1.2 & 1.8 & 1.8 & 1.7 & 1.6 \\
\hline
\end{tabular}

the table, using the max/dip and COG variations of the least-squares approach, the target ranges are estimated with average absolute range errors of 1.2 and $1.7 \mathrm{~cm}$, respectively. Matched filtering results in an average absolute range error of $0.8 \mathrm{~cm}$ which is much better than that obtained with the leastsquares approach. The greatest contribution to the range errors comes from targets which are incorrectly differentiated. If we average over only correctly differentiated targets, the average absolute range errors are reduced to $0.6,0.6$, and $0.7 \mathrm{~cm}$ for the max/dip and COG variations of least-squares and the matched filter approaches, respectively. Since these numbers are comparable, we may conclude that the superior range accuracy of matched filtering is mostly a consequence of its superior differentiation accuracy.

As for azimuth estimation, matched filtering results in an average absolute estimation error of $1.6^{\circ}$, which is the best among the approaches compared. Averaging the azimuth errors over only correctly differentiated targets does not result in significant changes. This is due to the fact that azimuth estimation is not dependent on correct differentiation.

Because of the sharpness of the scans for the cylindrical target around their peaks, azimuth estimation of cylinders is more accurate than that of other targets when the least-squares approach is used. On the other hand, angular localization of corners is less accurate since it is difficult to estimate with good accuracy the exact angular location of the relatively shallow central dip, especially with the max/dip variation of least-squares approach. The COG variation is, on the average, better than the max/dip variation in azimuth estimation due to the fact that COG based calculations average out the noise in the return signal intensities.

\section{Conclusion}

In this study, differentiation and localization of commonly encountered indoor features or targets such as plane, cylinder, edge and corner is 
achieved using an inexpensive infrared emitter and detector pair. Different approaches are compared in terms of correct target differentiation, and range and azimuth estimation accuracy. A typical application of the demonstrated system would be in mobile robotics in surveying an unknown environment composed of such features or targets. Many artificial environments fall into this category. We plan to test and evaluate the developed system on a small mobile robot in our laboratory for map building in a test room composed of the primitive target types considered in this study.

The accomplishment of this study is that even though the intensity patterns are highly dependent on target location, and this dependence cannot be represented by a simple relationship, we achieve position-invariant target differentiation. An average correct target differentiation rate of $97 \%$ over all target types is achieved and targets are localized within absolute range and azimuth errors of $0.8 \mathrm{~cm}$ and $1.6^{\circ}$, respectively. The method we propose is scalable in the sense that the accuracy can be increased by increasing the number of reference scans without increasing the computational cost.

In this paper, we have demonstrated target differentiation using four basic target types. However, based on the data we have collected, it seems possible to increase this number up to at least ten, provided the targets are not too similar. Current work investigates the deduction of not only the geometry but also the surface properties of the target from its intensity scan without knowing its location. Preliminary results indicate that the method of this paper can be applied to this case with little or no modification by treating the combination of a particular geometry and particular surface as a generalized target type. These results will be reported elsewhere [34].

\section{Acknowledgements}

This research was supported by TÜBITTAK under BDP and 197E051 grants. The authors would like to thank the Department of Engineering Science of the University of Oxford for donating the infrared sensors.

\section{References}

[1] P.J. Phillips, IEEE Trans. Image Process. 7 (8) (1998) 1150.

[2] L. Kwon, S.Z. Der, N.M. Nasrabadi, Opt. Eng. 41 (1) (2002) 69.

[3] T. Tsao, Z.Q. Wen, Opt. Eng. 41 (3) (2002) 697.

[4] I. Pavlidis, P. Symosek, B. Fritz, M. Bazakos, N. Papanikolopoulos, Mach. Vision Appl. 11 (6) (2000) 313.

[5] P.M. Tag, R.L. Bankert, L.R. Brody, J. Appl. Meteorol. 39 (2) (2000) 125.

[6] A.K. Jain, N.K. Ratha, S. Lakshmanan, Pattern Recognition 30 (2) (1997) 295.

[7] Z. Zalevsky, D. Mendlovic, E. Rivlin, S. Rotman, Opt. Eng. 39 (10) (2000) 2609.

[8] B. Bhanu, P. Symosek, S. Das, Pattern Recognition 30 (2) (1997) 197.

[9] F.T.S. Yu, S. Jutamulia (Eds.), Optical Pattern Recognition, Cambridge University Press, Cambridge, 1998.

[10] F.T.S. Yu, S. Yin (Eds.), Selected Papers on Optical Pattern Recognition, SPIE Milestone Series, vol. MS 156, SPIE Optical Engineering Press, Bellingham, Washington, 1999.

[11] D. Casasent, D. Psaltis, Opt. Commun. 17 (1) (1976) 59.

[12] M. McDonnell, Opt. Commun. 25 (3) (1978) 320.

[13] H.H. Arsenault, Y.N. Hsu, K. Chalasinska-Macukow, Opt. Eng. 23 (6) (1984) 705.

[14] F.T.S. Yu, X. Li, E. Tam, S. Jutamulia, D.A. Gregory, Appl. Opt. 28 (22) (1989) 4725.

[15] G. Gheen, Opt. Eng. 29 (9) (1990) 1029.

[16] C. Gu, J. Hong, S. Campbell, Opt. Commun. 88 (4-6) (1992) 309.

[17] A. Lohmann, Z. Zalevsky, D. Mendlovic, Opt. Commun. 128 (4-6) (1996) 199.

[18] P. Refregier, Opt. Commun. 86 (2) (1991) 113.

[19] E. Cheung, V.J. Lumelsky, IEEE Trans. Robot. Automat. 5 (6) (1989) 740.

[20] A.J. Hand, Photon. Spectra 32 (11) (1998) 30.

[21] H.C. Wikle, S. Kottilingam, R.H. Zee, B.A. Chin, J. Mater. Process. Technol. 113 (1-3) (2001) 228.

[22] B. Butkiewicz, Lect. Notes Comput. Sci. 1226 (1997) 74.

[23] V.J. Lumelsky, E. Cheung, IEEE Trans. Syst. Man Cybern. 23 (1) (1993) 194.

[24] A.M. Flynn, Int. J. Robot. Res. 7 (6) (1988) 5.

[25] P.M. Novotny, N.J. Ferrier, in: Proceedings of IEEE International Conference on Robotics and Automation, Detroit, MI, 10-15 May, 1999, p. 1644.

[26] F.J. Cuevas, M. Servin, R. Rodriguez-Vera, Opt. Commun. 163 (4-6) (1999) 270.

[27] P. Klysubun, G. Indebetouw, T. Kim, T.C. Poon, Opt. Commun. 184 (5-6) (2000) 357.

[28] J.J. Esteve-Taboada, P. Refregier, J. Garcia, C. Ferreira, Opt. Commun. 202 (1-3) (2002) 69. 
[29] K. Hashimoto, T. Tsuruta, K. Morinaka, N. Yoshiike, Sensors and Actuators A - Phys. 79 (1) (2000) 46.

[30] D.M. Scott, Meas. Sci. Technol. 6 (2) (1995) 156.

[31] Matrix Elektronik, AG, Kirchweg 24 CH-5422 Oberehrendingen, Switzerland, IRS-U-4A Proximity Switch Datasheet, 1995.
[32] Arrick Robotics, P.O. Box 1574, Hurst, Texas, 76053, RT12 Rotary Positioning Table, 2002, www.robotics.com/ rt12.html.

[33] J.W. Goodman, in: Introduction to Fourier Optics, second ed., McGraw-Hill, New York, 1996, p. 246.

[34] T. Aytaç, Master's Thesis, Bilkent University, Department of Electrical Engineering, Ankara, Turkey, 2002. 